\title{
Erhöht Adipositas auch das Risiko für sinunasale Entzündungen?
}

In einer US-Studie fielen unter den adipösen Probanden überdurchschnittlich viele Patienten mit allergischer Rhinitis und chronischer Rhinosinusitis auf.

A ktuelle Erkenntnisse lassen vermuten, dass sowohl die chronische Rhinosinusitis (CRS) und die allergische Rhinitis (AR) als auch die Adipositas mit entzündlichen Prozessen einhergehen. In einer amerikanischen Studie der Harvard Medical School wurde nun untersucht, ob zwischen krankhaftem Übergewicht und sinunasalen Entzündungen möglicherweise ein

Nasenprobleme?
Zusammenhang besteht. Mittels Querschnittsanalyse einer amerikanischen Panelbefragung aus den Jahren 2008 und 2010 wurden Erwachsene mit chronischer CRS und/oder AR erfasst und gleichzeitig das Körpergewicht der Personen ermittelt.

Das Ergebnis: 7,7\% der erwachsenen Amerikaner litten an einer allergischen Rhinitis, $5,7 \%$ an einer chronischen Rhinosinusitis. Fast ein Drittel (29\%) galt als adipös (BMI $\geq 30 \mathrm{~kg} / \mathrm{m} 2$ ). Mit zunehmendem Körpergewicht wurde der Zusammenhang zwischen AR bzw. CRS und Adipositas immer deutlicher. In der multivariaten Analyse lag bei den adipö- sen Probanden der Anteil der Personen mit allergischer Rhinitis um $22 \%$ und mit chronischer Rhinosinusitis um 31\% höher als in der gesamten Studienpopulation.

Fazit: Die aktuellen Daten lassen eine Assoziation zwischen Adipositas und allergischer Rhinitis bzw. chronischer Rhinosinusitis erkennen. Dabei ist der zugrunde liegende Mechanismus noch unklar. Möglicherweise, so die Autoren, spielen bestimmte Mediatoren aus dem Fettgewebe eine Rolle, die die sinunasale Entzündung begünstigen. Weitere Untersuchungen werden zeigen, ob mit den Pfunden auch der Schweregrad der Erkrankungen zunimmt bzw. ob eine Adipositas die Prognose sinunasaler Erkrankungen langfristig verschlechtert. Dr. Christine Starostzik

Bhattacharyya N. Associations between obesity and inflammatory sinonasal disorders. Laryngoscope 2013; Feb 9 [Epub ahead of print]

\section{Unauffälliges Neugeborenen-Screening - keine Versicherung gegen spätere Hörstörungen}

Auch wenn Kinder das universelle Neugeborenen-Hörscreening (UNHS) erfolgreich absolvieren, können im weiteren Verlauf Hörstörungen auftreten. HNO-Kollegen regen deshalb an, über zusätzliche Tests nachzudenken.

D ie Ärzte um Dr. Kavita Dedhia von der Universitätsklinik in Pittsburgh durchforsteten für ihre retrospektive Studie die Krankenakten der Kinderklinik aus den Jahren 2001 bis 2011. Sie konzentrierten sich dabei auf jene Kinder, die beim Neugeborenen-Hörscreening unauffällig waren und bei denen später Schwerhörigkeit diagnostiziert wurde.

Das Gesetz zum UNHS trat im US-Bundesstaat Pennsylvania 2001 in Kraft. Der erste Hörtest erfolgt nach der Geburt noch in der Klinik, ein weiterer bis zum Ende des ersten Lebensmonats. Ziel ist, wie in Deutschland, eine frühzeitige Therapie noch vor dem sechsten Lebensmonat.

Bei Durchsicht der Akten entdeckten Dedhia und ihre Kollegen fast 1.000 Kinder mit Schwerhörigkeit. 78 von diesen waren im UNHS unauffällig, zeigten aber später Symptome einer Schwerhörigkeit. Bei 28 Kindern dieser Gruppe hatten die Eltern den Verdacht auf eine Hörstö- rung, 25 weitere Kinder mit einer Hörstörung fielen erst bei einem Test in der Schule auf. Bei $17 \%$ der Kinder kam es zu Verzögerungen in der Sprachentwicklung, 12\% fielen bei einem Hörtest beim Hausarzt durch. Mit 37 Fällen hatte fast jedes zweite Kind eine starke oder sogar sehr starke Schwerhörigkeit.

Die Ärzte vermuten, dass einige dieser Kinder beim ersten Test als unauffällig durchgerutscht waren und dass sich bei anderen die Hörstörung erst mit der Zeit entwickelte. Immerhin bei jedem dritten Kind drängte sich den Eltern - unter anderem beim Vergleich mit eigenen oder fremden Kindern in der gleichen Altersgruppe - der Verdacht auf eine Hörstörung auf, und sie baten um einen erneuten Hörtest.

Die US-Ärzte erinnern daran, dass das UNHS auch zu falsch-negativen Ergebnissen führen kann. Als Beispiel nennen sie Kinder mit einer „auditory neu- ropathy spectrum disorder" (ANSD). Die Kleinen können den Hörtest bestehen, weil sie eigentlich normal hören. Allerdings sei die Worterkennung schlechter als bei gesunden Kindern, so die Ärzte. Auch könnten bei hohen Schallenergien akustische Reflexe bei ihnen fehlen oder aber verstärkt werden. Der Test auf otoakustische Emissionen ist dann normal, aber die Hirnstammaudiometrie auffällig. Ohne Durchführung einer Audiometrie werde bei diesen Kindern erst viel später die Diagnose "Schwerhörigkeit" gestellt, betonten die HNO-Ärzte.

Fazit: Ob weitere Tests helfen können, Kinder mit Hörstörungen besser zu erkennen, muss noch geprüft werden. Nicht zuletzt ist das auch eine Kostenfrage. An der Klinik in Pittsburgh wollen die Ärzte nun herausfinden, ob ein weiterer Hörtest beim üblichen DreiMonats-Check-up dazu beitragen kann, bisher nicht entdeckte Kinder mit Hörstörungen doch noch ausfindig zu machen.

Peter Leiner

Dedhia K. et al. Children with sensorineural hearing loss after passing the newborn hearing screen. JAMA Otolaryngol Head Neck Surg 2013; 139: 119-23 